\title{
Oral dextrose reduced procedural pain without altering cellular ATP metabolism in preterm neonates: a prospective randomized trial
}

\author{
Danilyn M. Angeles $\mathbb{1}^{1,2} \cdot$ Danilo S. Boskovic $\mathbb{1}^{1} \cdot$ John C. Tan ${ }^{1} \cdot$ Wendy Shih ${ }^{3} \cdot$ Erin $\mathrm{Hoch}^{4} \cdot$ Dorothy Forde $\mathbb{1}^{5} \cdot$ \\ Raylene M. Phillips $s^{2,4}$. Andrew Hopper $\mathbb{D}^{2,4}$ - Douglas D. Deming $\mathbb{D}^{2,4} \cdot$ Mitchell Goldstein $^{2,4}$. Giang Truong ${ }^{2,4}$. \\ Aprille Febre, ${ }^{2,4}$ Priscilla Pegis ${ }^{4}$ Adrian Lavery ${ }^{2,4} \cdot$ Munaf Kadri $^{2,4} \cdot$ Anamika Banerji $^{2,4} \cdot$ Iman Mousselli $^{1}$. \\ Vora Farha ${ }^{2,4} \cdot$ Elba Fayard $^{2,4}$
}

Received: 29 August 2019 / Revised: 30 January 2020 / Accepted: 12 February 2020 / Published online: 26 February 2020

(c) The Author(s) 2020. This article is published with open access

\begin{abstract}
Objective To examine the effects of $30 \%$ oral dextrose on biochemical markers of pain, adenosine triphosphate (ATP) degradation, and oxidative stress in preterm neonates experiencing a clinically required heel lance.

Study design Utilizing a prospective study design, preterm neonates that met study criteria $(n=169)$ were randomized to receive either (1) 30\% oral dextrose, (2) facilitated tucking, or (3) 30\% oral dextrose and facilitated tucking 2 min before heel lance. Plasma markers of ATP degradation (hypoxanthine, uric acid) and oxidative stress (allantoin) were measured before and after the heel lance. Pain was measured using the premature infant pain profile-revised (PIPP-R).

Results Oral dextrose, administered alone or with facilitated tucking, did not alter plasma markers of ATP utilization and oxidative stress.

Conclusion A single dose of $30 \%$ oral dextrose, given before a clinically required heel lance, decreased signs of pain without increasing ATP utilization and oxidative stress in premature neonates.
\end{abstract}

\section{Introduction}

Premature infants admitted to the neonatal intensive care unit (NICU) require up to several hundred procedures during their hospitalization [1]. Many of these are tissue-damaging procedures (TDPs) known to cause pain. It was previously shown that TDPs, such as tape removal, not only lead to signs of pain but also increase markers of adenosine triphosphate (ATP) degradation and oxidative stress [2]. Further investigations

Danilyn M. Angeles

dangeles@1lu.edu

1 Department of Basic Sciences, School of Medicine, Loma Linda University, Loma Linda, CA, USA

2 Department of Pediatrics, School of Medicine, Loma Linda University, Loma Linda, CA, USA

3 School of Public Health, Loma Linda Univeristy, Loma Linda, CA, USA

4 Loma Linda University Children's Hospital, Loma Linda, CA, USA

5 School of Nursing, University of California, San Francisco, CA, USA probed if interventions that appear to relieve pain also reduce biochemical markers of ATP degradation and oxidative stress. Oral sucrose is a commonly used analgesic that has been shown to significantly decrease pain scores when given prior to TDPs [3, 4], and was expected to also reduce markers of ATP degradation and oxidative stress. However, although $24 \%$ oral sucrose significantly reduced behavioral markers of pain, it significantly increased heart rate and biochemical markers of ATP degradation (hypoxanthine, uric acid) and oxidative stress (allantoin) over time [5].

Moreover, this effect was enhanced in neonates that were intubated or were receiving more than $30 \% \mathrm{FiO}_{2}$ [6]. It, therefore, became necessary to look for an intervention, or a combination of interventions, which would decrease both behavioral and physiological markers of procedural pain, and not increase the biochemical markers of ATP utilization and oxidative stress.

\section{Method}

The individual and additive effects of two commonly used interventions for procedural pain were tested. These 
interventions were (a) $30 \%$ oral dextrose (d-glucose), (b) facilitated tucking, and (c) a combination of $30 \%$ oral dextrose and facilitated tucking. Sweet solutions such as sucrose and dextrose are documented to reduce pain scores $[4,7,8]$. However, oral dextrose has the potential to decrease behavioral and physiological signs of pain while avoiding the metabolic costs of fructose, a key component of sucrose [9]. The adverse effects of oral sucrose include a reduction in ATP synthesis and phosphate depletion due to poorly regulated fructose metabolism $[9,10]$. Facilitated tucking is the gentle positioning of preterm infants with arms and legs in a flexed, midline position close to the body, while either in a side-lying or prone position [11]. Facilitated tucking has been documented to reduce the behavioral and physiological signs of pain, as evidenced by a reduction in pain scores and heart rate [11, 12]. This study's painful procedure is a clinically required heel lance, which refers to a puncture of the newborn's heel for a blood glucose test using a specially designed lancet.

A prospective randomized study was conducted at Loma Linda University Children's Hospital Neonatal Intensive Care Unit (LLUCH-NICU) from October 2014 to December 2018. The study protocol and informed consent documents were approved by the Loma Linda University Health Institutional Review Board. Families were approached for consent as soon as possible after birth. Figure 1 describes the study inclusion and exclusion criteria and the flowchart of subject enrollment. Subjects were premature infants born at $>24$ weeks gestational age (GA) who had an arterial or central catheter in place and clinically required heel lancing at 27-36 weeks corrected GA. Exclusion criteria included (1) requirement for surgery, (2) intraventricular hemorrhage (IVH) $\geq$ grade 3 , (3) neonates on medications such as morphine, fentanyl, midazolam, muscle relaxants, phenobarbital, or phenytoin, (4) renal injury (plasma creatinine $>1 \mathrm{mg} / \mathrm{dl}$ ), (5) cyanotic heart lesions requiring inotropic support, (6) respiratory distress (increased work of breathing as evidenced by tachypnea, nasal flaring, chest retractions and grunting [13], (7) chromosomal anomalies, and (8) facial anomalies. To minimize the risk for hypovolemia, potential subjects were excluded if study sampling (along with clinically-related blood sampling) could potentially result in blood volume loss of more than $10 \%$. Parents of premature infants who met the study criteria were approached for informed consent soon after birth. Once consent was obtained, subjects were randomized as described in Fig. 2. Randomization was performed by the research pharmacist using randomization tables generated by our statistician.

\section{Sample size determination}

The sample size for the study was determined based on statistical power to detect changes in outcomes for the three intervention groups across time. Using a repeated-measures ANOVA (i.e., $F$ test) based on a Type-I error rate of 5\%, a total of 120 subjects (40 in each group) are needed to detect a small to moderate effect size of 0.25 (changes in pain scores and biochemical marker concentration) with $80 \%$ power. After accounting for an anticipated $10 \%$ attrition rate, a total of 132 subjects (44 in each group) would be needed.

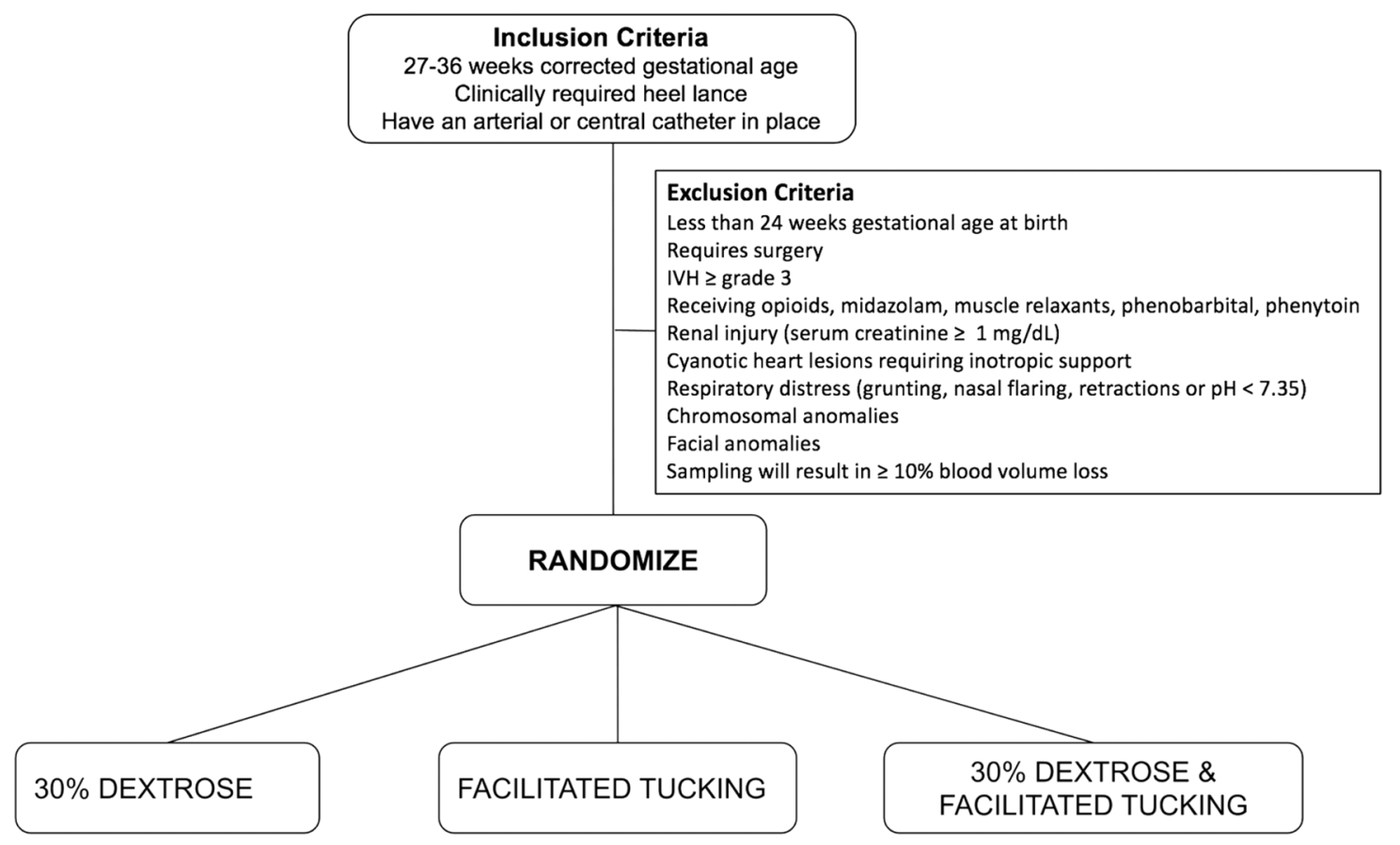

Fig. 1 Study inclusion and exclusion criteria and experimental groups. Randomization procedure. 
Fig. 2 Flowchart of subject enrollment, randomization and analysis. Enrollment layout.

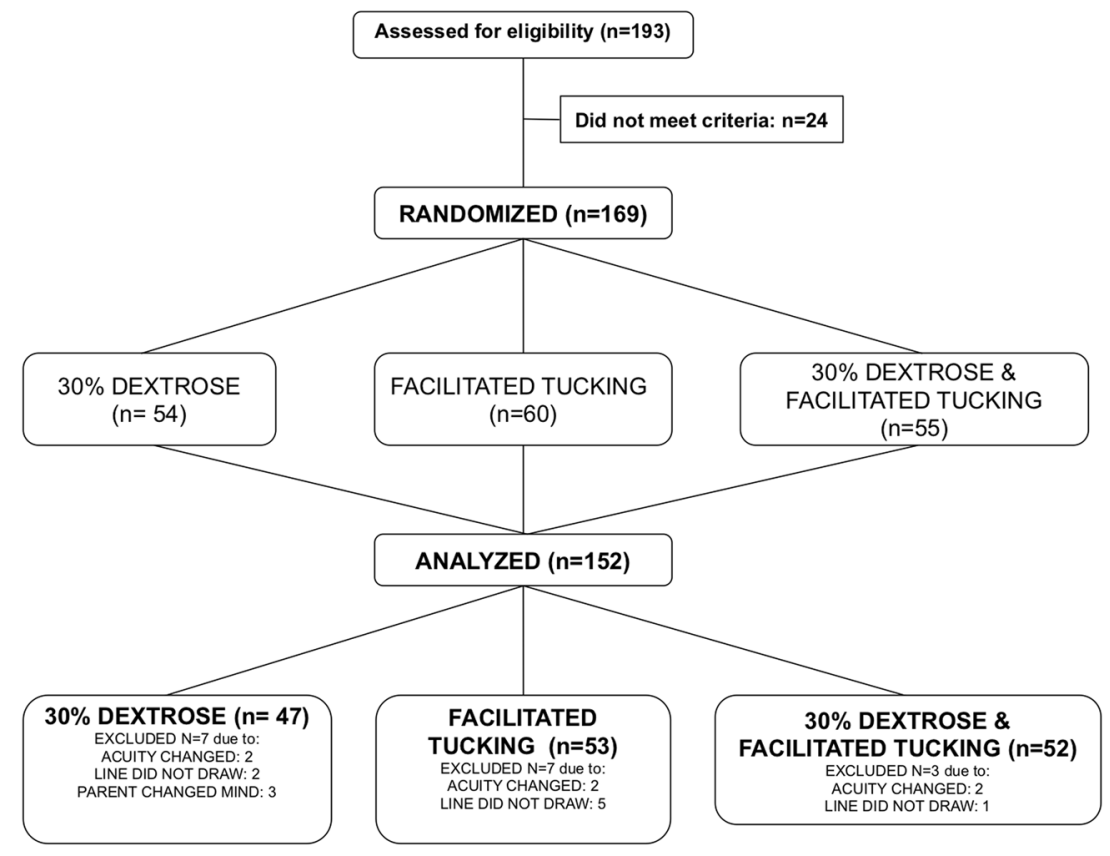

The flow of the study procedure is described in Fig. 3. Pain scores were measured at baseline $(0 \mathrm{~min})$ and at the time of the heel lance, and were determined on a scale of 0 through 21 using the premature infant pain profile-revised (PIPP-R) [14]. Biochemical plasma markers were measured at baseline $(0 \mathrm{~min})$ and $5 \mathrm{~min}$ after the heel lance. The "5 min" time period was chosen based on prior studies, demonstrating significant increases in plasma purines $5 \mathrm{~min}$ after heel lance, followed by purine levels below baseline 15-30 min after heel lance [5]. The dose of 30\% dextrose, $0.5 \mathrm{~mL} / \mathrm{Kg}$ was selected based on published studies on premature infants, showing this dose to be most effective in reducing signs of pain [8,15-17]. Oral dextrose was prepared by our clinical pharmacist and was administered over $60 \mathrm{~s}$ via syringe to the anterior tongue $2 \mathrm{~min}$ prior to the heel lance followed by giving the subject a pacifier.

\section{Assessment of pain}

We used the PIPP-R [14], an instrument that was designed to assess acute pain in preterm neonates. This tool has a physiological component that scores changes in oxygen saturation and heart rate as well as a behavioral component comprising facial expressions and behavioral state. Changes in facial expression include brow lowering or brow bulge, eye squeeze, and nasolabial furrowing. The facial expression score is based on the duration of observed expression (the longer the duration of brow bulge, the higher the score, up to a maximum score of 3 for each facial expression). A score of 6 or below indicates an absence of pain, while a score of 12 and above indicates moderate to severe pain [14].

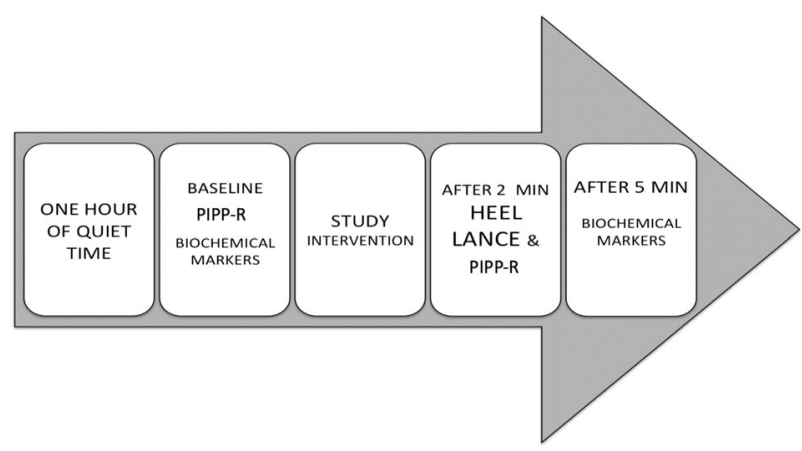

Fig. 3 Data collection strategy and approach. Study procedure.

\section{Measurement of biochemical markers of hypoxia, oxidative stress, and oxidative cell injury in plasma}

Plasma markers were measured at baseline ("0" min) and $5 \mathrm{~min}$ after completion of heel lance. A total of $1.6 \mathrm{~mL}$ of blood $(0.8 \mathrm{~mL}$ for each sampling period) was collected from an arterial or central catheter and placed in cooled plastic vials containing EDTA. Blood samples were centrifuged within 5 min to separate the plasma, which was stored at $-80^{\circ} \mathrm{C}$. All samples were analyzed immediately or within 1 week of acquisition.

\section{Measurement of purines}

Purine metabolites (hypoxanthine and uric acid) were measured as previously published by our laboratory $[2,5,6]$. Specifically, plasma was removed, transferred to separate Eppendorf tubes, and immediately centrifuged in Eppendorf 
5702R (Pittsburgh, Pennsylvania) centrifuge, for $30 \mathrm{~min}$ at $18,000 \times g$. The supernatant was transferred to Microcon centrifugal filter devices (Millipore Corp, Bedford, Massachusetts), $200 \mu \mathrm{L}$ per device, and spun for $90 \mathrm{~min}$ at $14,000 \times g, 4{ }^{\circ} \mathrm{C}$. The filtrate was removed, and $150 \mu \mathrm{L}$ was transferred to an Eppendorf tube containing $1 \times 10^{-7} \mathrm{~mol}$ of 2-aminopurine (internal standard). High-performance liquid chromatography (HPLC) (Waters 996 PDA, 715 Ultra Wisp Sample Processor; Millipore Corp) analysis was performed on the same day, or the tubes were frozen at $-80^{\circ} \mathrm{C}$ until analysis. Previous HPLC analysis of plasma demonstrated that purines remained stable with freezing [2, 5].

Three $45 \mu \mathrm{L}$ injections were used for each sample onto a Supelcosil LC-18-S $15 \mathrm{~cm} \times 4.6 \mathrm{~mm}, 5 \mu \mathrm{m}$ column (SGE, Austin, Texas), with the following isocratic conditions: $50 \mathrm{mM}$ ammonium formate buffer, $\mathrm{pH} 5.5$, flow rate $1.0 \mathrm{~mL} / \mathrm{min}$. Hypoxanthine and uric acid were quantified by obtaining peak areas at appropriate retention times and wavelengths $[5,18]$. Once the peak area of 2-aminopurine at $\sim 10.8 \mathrm{~min}$ and $305 \mathrm{~nm}$ was determined, the area ratios of hypoxanthine and uric acid to 2-aminopurine were determined and converted to micromolar concentrations using standard curves. Samples were analyzed in triplicates, and values with coefficients of variation of $<10 \%$ were included in the final analyses. The limits of detection for the purines are as follows: $1.58 \mu \mathrm{M}$ hypoxanthine and $5.0 \mu \mathrm{M}$ uric acid. The research technician that performed purine analysis was blinded to group assignment.

\section{Measurement of allantoin}

Allantoin was measured in plasma as previously published by our laboratory $[2,5,6]$ using an adaptation of the methods developed by Gruber et al. [19] and Pavitt et al. [20]. Plasma $(50 \mu \mathrm{L})$ was transferred to an Eppendorf tube containing $5 \times$ $10^{-10}$ mol internal standard $\left(50 \mu \mathrm{L} \quad 10 \mu \mathrm{M} \quad\left[{ }^{15} \mathrm{~N}\right]\right.$-labeled allantoin). Spiked samples were simultaneously deproteinized and extracted by the addition of $100 \mu \mathrm{L}$ of acetonitrile. These samples were then vortexed and centrifuged at $20,000 \times \mathrm{g}$, $4{ }^{\circ} \mathrm{C}$ for $5 \mathrm{~min}$, and the supernatant was dried under $\mathrm{N}_{2}$. After drying, $50 \mu \mathrm{L}$ of MTBSTFA (i.e., N-methyl-N-tert-butyldimethylsilyltrifluoroacetamide) in pyridine (1:1 vol/vol) was added, and the derivatization reaction was facilitated by incubation at $50{ }^{\circ} \mathrm{C}$ for $2 \mathrm{~h}$. The analysis was performed on Agilent 6890N Network GC System connected to an Agilent 5973 Inert Mass Selective Detector (both Agilent Technologies, Inc, Santa Clara, California). The separation was performed using an Agilent 122-5532G capillary column (25.7 $\mathrm{m}$ length, $0.25 \mathrm{~mm}$ internal diameter). Helium was used as the carrier gas at a flow rate of $1.5 \mathrm{~mL} / \mathrm{min}$. Derivatized product $(1 \mu \mathrm{L})$ was injected in split mode (split 20:1, split flow $29.4 \mathrm{~mL} / \mathrm{min}$, total flow $33.8 \mathrm{~mL} / \mathrm{min}$ ). The initial column temperature was set at $100{ }^{\circ} \mathrm{C}$ and held at that temperature for $2 \mathrm{~min}$; it was increased to $180{ }^{\circ} \mathrm{C}$ at a rate of $10^{\circ} \mathrm{C} / \mathrm{min}$. The column was held at this temperature for $4 \mathrm{~min}$ and then increased to $260^{\circ} \mathrm{C}$ at a rate of $20^{\circ} \mathrm{C} / \mathrm{min}$. This temperature was maintained until the end of the run. Allantoin was quantified using selected ion monitoring mode with the $398.00 \mathrm{~m} / \mathrm{z}$ ion monitored for allantoin and the $400.00 \mathrm{~m} / \mathrm{z}$ for DL-allantoin-5- ${ }^{13} \mathrm{C} ; 1-{ }^{15} \mathrm{~N}$. The ion abundance ratios (398.00/ 400.00) were converted to micromolar concentrations by the use of a standard curve. The research technician that performed allantoin analysis was blinded to group assignment.

\section{Statistics}

Clinical and demographic characteristics were stratified by intervention group, summarized as count and percentages (\%) for categorical characteristics, and mean and standard deviation (SD) for continuous characteristics. Categorical variables were compared with intervention groups using a $\chi^{2}$ test and continuous variables using a $t$-test.

Repeated-measures ANOVA was utilized to evaluate the effects of interventions on plasma purines and allantoin concentrations over time, with main effects of intervention group, time, and intervention group by time interactions. Separate models were fit for each outcome. The intervention effect was defined as a significant interaction effect between intervention group and time. Correlations between purines, allantoin, and biobehavioral markers (i.e., PIPP-R) were examined using Spearman's rho. Statistical analyses for this study were generated using SAS software (version 9.4). Statistical significance was defined as $p<0.05$.

\section{Results}

\section{Subject demographics}

As shown in Fig. 2, we obtained consent from 169 subjects who were randomized into three groups to receive (a) $30 \%$ oral dextrose alone: $n=54$, (b) facilitated tucking alone: $n=60$ or (c) $30 \%$ oral dextrose and facilitated tucking: $n=$ 55 , respectively, 2 min before a clinically required heel lance. Seventeen subjects were excluded after randomization due to reasons described in Fig. 2. As shown in Table 1, there were no significant differences in birth weight, GA, Apgar scores, and acuity scores (SNAPPE-II) between the three groups.

\section{Effect of $30 \%$ dextrose with and without facilitated tucking on behavioral and physiological markers of pain}

There were no significant differences in baseline pain scores between the three intervention groups (Table 2). There were 
Table 1 Subject demographics and clinical characteristics.

\begin{tabular}{llll}
\hline Subject characteristics & $\begin{array}{l}30 \% \text { dextrose } \\
n=47\end{array}$ & $\begin{array}{l}\text { Facilitated tucking } \\
n=53\end{array}$ & $\begin{array}{l}30 \% \text { dextrose and facilitated tucking } \\
n=52\end{array}$ \\
\hline EGA at birth (weeks) & $28^{4 / 7} \pm 3^{2 / 7}$ & $29^{1 / 7} \pm 2^{6 / 7}$ & $29^{2 / 7} \pm 2^{4 / 7}$ \\
EGA at time of study procedure (weeks) & $31^{2 / 7} \pm 2^{3 / 7}$ & $31^{1 / 7} \pm 4^{3 / 7}$ & $31^{3 / 7} \pm 2^{1 / 7}$ \\
Gender & Male: $30(64 \%)$ & Male: $35(66 \%)$ & Male: $28(54 \%)$ \\
& Female: $17(36 \%)$ & Female: $18(34 \%)$ & Female: $24(46 \%)$ \\
Apgar score, 0 min median (min, max) & $5(1,9)$ & $4(0,9)$ & $5(1,9)$ \\
Apgar score, 5 min median (min, max) & $8(1,9)$ & $8(1,9)$ & $8(2,10)$ \\
SNAPPE-II at time of study procedure & $20.9 \pm 16.2$ & $21.2 \pm 16.0$ & $16.3 \pm 14.0$ \\
Mode of oxygen delivery at time of study & Spontaneous RA: 10 & Spontaneous RA: 9 & Spontaneous RA: 6 \\
procedure & HFNC: 5 & HFNC: 12 & HFNC: 12 \\
& NCPAP: 23 & NCPAP: 27 & NCPAP: 25 \\
& NIPPV: 3 & NIPPV: 3 & NIPPV: 6 \\
& SIMV: 5 & SIMV: 0 & SIMV: 3 \\
& HFV: 1 & HFV: 2 & HFV: 0 \\
\hline
\end{tabular}
physiological, and biochemical signs of pain, ATP utilization, and oxidative stress.
Table 2 Behavioral,

\begin{tabular}{|c|c|c|c|c|}
\hline Characteristics & $\begin{array}{l}30 \% \text { dextrose } \\
n=47\end{array}$ & $\begin{array}{l}\text { Facilitated } \\
\text { tucking } \\
n=53\end{array}$ & $\begin{array}{l}30 \% \text { dextrose and } \\
\text { facilitated tucking } \\
n=52\end{array}$ & $P$ value* \\
\hline \multicolumn{5}{|l|}{ PIPP-R: median (min, max) } \\
\hline Baseline & $5(0,17)$ & $5(0,14)$ & $6(0,13)$ & 0.124 \\
\hline Heel lance & $7(0,15)$ & $8(0,17)$ & $8(0,16)$ & 0.783 \\
\hline \multicolumn{5}{|l|}{ Heart rate, bpm } \\
\hline \multicolumn{5}{|l|}{ Mean \pm SD } \\
\hline Baseline & $162.78 \pm 12.63$ & $160.90 \pm 19.10$ & $167.02 \pm 14.41$ & 0.159 \\
\hline Heel lance & $165.02 \pm 13.73$ & $165.08 \pm 13.34$ & $162.70 \pm 29.06$ & 0.965 \\
\hline $\begin{array}{l}\text { Minimum } \mathrm{O}_{2} \text { sat during heel } \\
\text { lance }(\%) \text {, mean } \pm \mathrm{SD}\end{array}$ & $94.44 \pm 4.69$ & $91.33 \pm 7.28$ & $92.10 \pm 5.77$ & 0.052 \\
\hline \multicolumn{5}{|l|}{ Uric acid } \\
\hline Baseline & $145.82(70.96)$ & $119.84(51.14)$ & $136.57(64.94)$ & 0.08 \\
\hline $5 \mathrm{~min}$ & $140.76(73.11)$ & $120.4(49.48)$ & $133.56(65.72)$ & 0.201 \\
\hline \multicolumn{5}{|l|}{ Hypoxanthine } \\
\hline Baseline & $1.35(1.35)$ & $1.35(2.09)$ & $1.37(1.21)$ & 0.739 \\
\hline $5 \mathrm{~min}$ & $1.33(1.29)$ & $1.44(2.17)$ & $1.28(1.13)$ & 0.927 \\
\hline \multicolumn{5}{|l|}{ Allantoin } \\
\hline Baseline & $29.24(18.29)$ & $31.95(24.17)$ & $26.31(13.83)$ & 0.642 \\
\hline $5 \mathrm{~min}$ & $27.83(16.68)$ & $30.72(21.13)$ & $26.78(14.84)$ & 0.75 \\
\hline
\end{tabular}

*Bivariate comparisons.

also no significant differences in pain scores in response to heel lance between the three groups (Table 2). During the heel lance, the median PIPP-R score of subjects in the dextrose only group was 7 (min-max: 0,15), median PIPP$\mathrm{R}$ score of subjects in the facilitated tucking only group was 8 (min-max: 0, 17), and median PIPP-R score of subjects in the combination dextrose and facilitated tucking group was 8 (min-max: 0,16), $p=0.783$ ) (Table 2). Heart rate and oxygen saturation during heel lance were not significantly different between the three groups from baseline to heel lance ( $p=0.276$ and $p=0.163$, respectively) (Table 2).
Effects of $30 \%$ dextrose with and without facilitated tucking on markers of ATP metabolism (purines) and oxidative stress (allantoin)

Oral dextrose with and without facilitated tucking did not increase plasma markers of ATP metabolism and oxidative stress (Table 2). Mean concentrations of hypoxanthine and uric acid decreased slightly over time or did not change significantly from baseline (Table $2, p=0.98$ and $p=0.07)$. We also found no significant increase in allantoin across the three intervention groups $(p=0.266)$, 
even among neonates with minimal pain response to heel lance.

\section{Discussion}

We found that the use of $30 \%$ oral dextrose, with or without facilitated tucking, 2 min before a heel lance had no significant effect on plasma markers of ATP utilization and oxidative stress. This finding is in contrast to the effects of using $24 \%$ sucrose (SweetEase ${ }^{\mathrm{TM}}$ ), which was shown to increase cellular energy utilization and oxidative stress $[5,6]$. The difference may be due to the metabolism of the fructose moiety of sucrose. Although fructose and dextrose (d-glucose) have identical chemical formulas, their chemical structure is dissimilar, resulting in distinctions in absorption and metabolism. Unlike fructose, the metabolism of glucose is highly regulated and dependent on cellular energy demand. If the cellular need for energy is modest, glucose influx is lessened through a reduction in the density of glucose transporters (GLUT2) at the plasma membrane, and glucose is diverted away from glycolysis through allosteric inhibition of phosphofructokinase-1 [10].

In contrast, the cellular influx and metabolism of fructose occurs largely independently of cell's energy needs, leading to substrate-dependent phosphate depletion, decreased ATP synthesis, and uric acid production $[9,21]$. This biochemical reaction has been documented in the hepatocytes of children and adults [22]. It was also shown in premature neonates, where a single dose of oral sucrose given before a clinically required heel lance increased plasma markers of increased ATP degradation $[5,6]$. These findings suggested that administration of oral sucrose may increase ATP utilization due to the biochemical cost of metabolizing fructose. Our current data suggest that the administration of oral dextrose does not have this effect on ATP utilization.

More recently, the effect of fructose on glucose metabolism was investigated in adipocytes from human Simpson-Golabi-Behmel syndrome [23]. In the presence of $5 \mathrm{mM}$ glucose representing normal blood glucose concentration, fructose was shown to divert glucose metabolism away from glycolysis and oxidative phosphorylation, reducing ATP synthesis [23]. Glucose metabolism was also shown to be diverted away from glycogenesis, gluconeogenesis, ribose-phosphate synthesis, and nucleotide synthesis [23]. Instead, glucose was metabolized to lactate and diverted to serine oxidation glycine cleavage pathway (SOGC), a pathway utilized for lipogenesis and storage [23]. The diversion of glucose to the SOGC pathway resulted in lower ATP synthesis due to diminished mitochondrial energy metabolism [23]. It will be important to determine if this effect occurs in other cell types since fructose is also taken up and metabolized by skeletal muscle cells and renal cortical cells [24-26].

Presented results show that administration of $30 \%$ oral dextrose did not increase plasma allantoin levels, suggesting that oral dextrose has no significant effect on reactive oxygen species (ROS) formation. This finding is different from that previously observed using sucrose. A single dose of oral sucrose, given before a clinically required heel lance, significantly increased plasma allantoin concentration over time, in neonates whose pain increased minimally $(<33 \%$ increase in PIPP scores) in response to the heel lance. Formation of ROS may be due to fructose-induced hyperuricemia. While uric acid is documented to be an antioxidant, it can also act as a potent prooxidant molecule [25]. However, ROS can also be formed when accessible carbonyls of aldehydes or ketones interact with basic amino groups of proteins, or with free hydroxyls found in lipids [27]. At normal $\mathrm{pH}$ and temperature, glucose molecules are found in the stable 6-member glucopyranose ring form, limiting aldehyde exposure, and reducing ROS generation [27]. However, fructose forms a 5-member fructofuranose ring with two axial hydroxymethyl groups, exposing reactive ketone moiety [27]. This fructose-generated ROS can lead to cellular damage, especially in the liver, as has been demonstrated in cultured hepatocytes [28] and in animal models [29].

Heel lance was documented to result in moderate pain, with pain scores ranging from 8.5 to 11.2 (PIPP) in neonates given sterile water and pacifier [4, 8, 30]. We showed that $30 \%$ oral dextrose with and without facilitated tucking can reduce the pain score by a minimum of 2-3 points (Table 2). However, the difference in pain scores between the three intervention groups was not statistically significant, suggesting that facilitated tucking with nonnutritive sucking may be as effective as $30 \%$ oral dextrose in decreasing signs of pain. This is especially important because the long-term effects of repeated administration of sweet solutions like sucrose and dextrose, specifically on organs such as the brain, liver, kidneys, and skeletal muscle, are still unclear. A study by Johnston et al. showed that repeated sucrose administration effectively decreased pain scores, but higher doses of sucrose ( $>10$ doses per day) were associated with lower scores for motor development, vigor, alertness, and orientation at 36 and 40 weeks gestation [31]. It is not known whether repeated doses of $30 \%$ oral dextrose have similar effects. In addition, a recent study in neonatal mice showed that repeated exposure to sucrose led to significantly smaller white matter volumes (corpus callosum, stria terminalis, and fimbria) as well as a significantly smaller hippocampus and cerebellum [32]. Mice pups that received ten daily doses of sucrose, with nonpainful handling, had significantly poorer short-term memory in adulthood [33]. 
Moreover, the effect of sucrose or dextrose on the gastrointestinal system, starting with the mouth, has not been investigated. Does the administration of sweet solutions for pain alter the oral and intestinal microbiome of the premature infant? Because hospitalized neonates are exposed to 7-17 painful procedures per day [1], leading to potential administration of repeated doses of oral sucrose or dextrose per day, it is important to examine the effect of oral sucrose or oral dextrose on the development of the gastrointestinal microbiome.

This study's limitations are largely due to subjects' GAs, illness severity, and treatment dosage. (1) The mean corrected GA of subjects at the time of study was $31 \pm 2$ weeks, making the findings not generalizable to neonates that are $<29$ weeks gestation. (2) Enrolled subjects were clinically stable, as demonstrated by the mean SNAPPE-II score of 17-21, to reduce the effects of illness or tissue hypoxia on purine and allantoin levels. This reduces the applicability of findings to critically ill preterm neonates, considered to have SNAPPE-II scores of 37 and above [34]. An appropriately effective analgesic for critically ill preterm neonates is still not known. (3) This study examined the effects of a single dose of $30 \%$ oral dextrose with and without facilitated tucking. Effects of prolonged repeated doses of dextrose on ATP metabolism, oxidative stress, and pain relief are unknown. In addition, effects of sweet solutions on other biochemical markers, such as markers of inflammation, are not known. Further prospective, randomized controlled trials that examine short and long-term effects of $30 \%$ oral dextrose are needed.

\section{Conclusions}

Our findings show that $30 \%$ dextrose is an effective analgesic for preterm neonates experiencing heel lance. Because 30\% dextrose, with or without facilitated tucking, decreased signs of pain without altering cellular energy metabolism, it may be a better nonpharmacologic intervention for procedural pain in premature neonates than interventions containing fructose (such as sucrose). Longterm cellular and organ effects of sweet solutions given during the neonatal period are not yet well understood in humans. Further studies are required to investigate these ubiquitously administered analgesics.

Acknowledgements We thank Yayesh Asmerom, MS, for performing all the purine and allantoin measurements and Desiree Wallace, PharmD, for providing the study drug and assisting with the randomization of subjects. We also thank the NICU nurses at Loma Linda University Children's Hospital for their support of this project.

Funding This work was supported by NIH grant RO1 NR011209 (Angeles).
Author contributions All authors provided final approval of the submitted final version of the paper. DA designed the experiments, acquired the funding, participated in the analysis and interpretation of the data, wrote the original and final draft, and is accountable for all aspects of the study. EF, RP assisted in the design of the study and acquisition of data, participated in the interpretation of the data, made substantial contributions to the paper, and are accountable for all aspects of the study. DB and WS participated in the interpretation of the data, made substantial contributions to the writing of the paper, and are accountable for all aspects of the study. WS participated in the analysis and interpretation of the data and made substantial contributions to the writing of the paper and is accountable for all aspects of the study. EH, AH, DD, MG, GT, AF, AL, MK, AB, VF, PP, JT, DF, and IM participated in the acquisition and interpretation of the data, made substantial contributions to the writing of the paper and are accountable for all aspects of the study.

\section{Compliance with ethical standards}

Conflict of interest The authors declare that they have no conflict of interest.

Publisher's note Springer Nature remains neutral with regard to jurisdictional claims in published maps and institutional affiliations.

Open Access This article is licensed under a Creative Commons Attribution 4.0 International License, which permits use, sharing, adaptation, distribution and reproduction in any medium or format, as long as you give appropriate credit to the original author(s) and the source, provide a link to the Creative Commons licence, and indicate if changes were made. The images or other third party material in this article are included in the article's Creative Commons licence, unless indicated otherwise in a credit line to the material. If material is not included in the article's Creative Commons licence and your intended use is not permitted by statutory regulation or exceeds the permitted use, you will need to obtain permission directly from the copyright holder. To view a copy of this licence, visit http://creativecommons. org/licenses/by/4.0/.

\section{References}

1. Cruz MD, Fernandes AM, Oliveira CR. Epidemiology of painful procedures performed in neonates: a systematic review of observational studies. Eur J Pain. 2016;20:489-98.

2. Slater L, Asmerom Y, Boskovic DS, Bahjri K, Plank MS, Angeles $\mathrm{KR}$, et al. Procedural pain and oxidative stress in premature neonates. J Pain. 2012;13:590-7.

3. Kristoffersen L, Malahleha M, Duze Z, Tegnander E, Kapongo N, Stoen $\mathrm{R}$, et al. Randomised controlled trial showed that neonates received better pain relief from a higher dose of sucrose during venepuncture. Acta Paediatr. 2018;107:2071-8.

4. Stevens B, Yamada J, Ohlsson A, Haliburton S, Shorkey A. Sucrose for analgesia in newborn infants undergoing painful procedures. Cochrane Database Syst Rev. 2016;7:CD001069.

5. Asmerom Y, Slater L, Boskovic DS, Bahjri K, Holden MS, Phillips R, et al. Oral sucrose for heel lance increases adenosine triphosphate use and oxidative stress in preterm neonates. $\mathrm{J}$ Pediatr. 2013;163:29-35.e1.

6. Angeles DM, Asmerom Y, Boskovic DS, Slater L, Bacot-Carter $\mathrm{S}$, Bahjri $\mathrm{K}$, et al. Oral sucrose for heel lance enhances adenosine triphosphate use in preterm neonates with respiratory distress. SAGE Open Med. 2015;3:2050312115611431. 
7. Dilen B, Elseviers M. Oral glucose solution as pain relief in newborns: results of a clinical trial. Birth. 2010;37:98-105.

8. Bueno M, Yamada J, Harrison D, Khan S, Ohlsson A, AdamsWebber T, et al. A systematic review and meta-analyses of nonsucrose sweet solutions for pain relief in neonates. Pain Res Manag. 2013;18:153-61.

9. Mayes PA. Intermediary metabolism of fructose. Am J Clin Nutr. 1993;58 5 Suppl:754S-65S.

10. Liemburg-Apers DC, Imamura H, Forkink M, Nooteboom M, Swarts HG, Brock R, et al. Quantitative glucose and ATP sensing in mammalian cells. Pharm Res. 2011;28:2745-57.

11. Sundaram B, Shrivastava S, Pandian JS, Singh VP. Facilitated tucking on pain in pre-term newborns during neonatal intensive care: a single blinded randomized controlled cross-over pilot trial. J Pediatr Rehabil Med. 2013;6:19-27.

12. Obeidat H, Kahalaf I, Callister LC, Froelicher ES. Use of facilitated tucking for nonpharmacological pain management in preterm infants: a systematic review. J Perinat Neonatal Nurs. 2009;23:372-7.

13. Reuter S, Moser C, Baack M. Respiratory distress in the newborn. Pediatr Rev. 2014;35:417-28. quiz 29

14. Gibbins S, Stevens BJ, Yamada J, Dionne K, Campbell-Yeo M, Lee $\mathrm{G}$, et al. Validation of the premature infant pain profilerevised (PIPP-R). Early Hum Dev. 2014;90:189-93.

15. Freire NB, Garcia JB, Lamy ZC. Evaluation of analgesic effect of skin-to-skin contact compared to oral glucose in preterm neonates. Pain. 2008;139:28-33.

16. Skogsdal Y, Eriksson M, Schollin J. Analgesia in newborns given oral glucose. Acta Paediatr. 1997;86:217-20.

17. Gradin M, Eriksson M, Holmqvist G, Holstein A, Schollin J. Pain reduction at venipuncture in newborns: oral glucose compared with local anesthetic cream. Pediatrics. 2002;110:1053-7.

18. Plank MS, Calderon TC, Asmerom Y, Boskovic DS, Angeles DM. Biochemical measurement of neonatal hypoxia. J Vis Exp. 2011;54:2948.1-7.

19. Gruber J, Tang SY, Jenner AM, Mudway I, Blomberg A, Behndig A, et al. Allantoin in human plasma, serum, and nasal-lining fluids as a biomarker of oxidative stress: avoiding artifacts and establishing real in vivo concentrations. Antioxid Redox Signal. 2009;11:1767-76.

20. Pavitt DV, de Fonseka S, Al-Khalaf N, Cam JM, Reaveley DA. Assay of serum allantoin in humans by gas chromatography-mass spectrometry. Clin Chim Acta. 2002;318:63-70.

21. Perheentupa J, Raivio K. Fructose-induced hyperuricaemia. Lancet. 1967;2:528-31.

22. Mosca A, Nobili V, De Vito R, Crudele A, Scorletti E, Villani A, et al. Serum uric acid concentrations and fructose consumption are independently associated with NASH in children and adolescents. J Hepatol. 2017;66:1031-6.

23. Varma V, Boros LG, Nolen GT, Chang CW, Wabitsch M, Beger $\mathrm{RD}$, et al. Fructose alters intermediary metabolism of glucose in human adipocytes and diverts glucose to serine oxidation in the one-carbon cycle energy producing pathway. Metabolites. 2015;5:364-85.

24. Jaiswal N, Maurya CK, Arha D, Avisetti DR, Prathapan A, Raj $\mathrm{PS}$, et al. Fructose induces mitochondrial dysfunction and triggers apoptosis in skeletal muscle cells by provoking oxidative stress. Apoptosis. 2015;20:930-47.

25. Cirillo P, Gersch MS, Mu W, Scherer PM, Kim KM, Gesualdo L, et al. Ketohexokinase-dependent metabolism of fructose induces proinflammatory mediators in proximal tubular cells. J Am Soc Nephrol. 2009;20:545-53.

26. Andres-Hernando A, Li N, Cicerchi C, Inaba S, Chen W, RoncalJimenez C, et al. Protective role of fructokinase blockade in the pathogenesis of acute kidney injury in mice. Nat Commun. 2017;8:14181.

27. Lustig RH. Fructose: it's "alcohol without the buzz". Adv Nutr. 2013;4:226-35.

28. Lee O, Bruce WR, Dong Q, Bruce J, Mehta R, O'Brien PJ. Fructose and carbonyl metabolites as endogenous toxins. Chem Biol Interact. 2009;178:332-9.

29. Pickens MK, Yan JS, Ng RK, Ogata H, Grenert JP, Beysen C, et al. Dietary sucrose is essential to the development of liver injury in the methionine-choline-deficient model of steatohepatitis. J Lipid Res. 2009;50:2072-82.

30. Gibbins S, Stevens B, Hodnett E, Pinelli J, Ohlsson A, Darlington G. Efficacy and safety of sucrose for procedural pain relief in preterm and term neonates. Nurs Res. 2002;51:375-82.

31. Johnston CC, Filion F, Snider L, Majnemer A, Limperopoulos C, Walker $\mathrm{CD}$, et al. Routine sucrose analgesia during the first week of life in neonates younger than 31 weeks' postconceptional age. Pediatrics. 2002;110:523-8.

32. Tremblay S, Ranger M, Chau CMY, Ellegood J, Lerch JP, Holsti $\mathrm{L}$, et al. Repeated exposure to sucrose for procedural pain in mouse pups leads to long-term widespread brain alterations. Pain. 2017;158:1586-98.

33. Ranger M, Tremblay S, Chau CMY, Holsti L, Grunau RE, Goldowitz D. Adverse behavioral changes in adult mice following neonatal repeated exposure to pain and sucrose. Front Psychol. 2018;9:2394.

34. Harsha SS, Archana BR. SNAPPE-II (Score for Neonatal Acute Physiology with Perinatal Extension-II) in predicting mortality and morbidity in NICU. J Clin Diagn Res. 2015;9:SC10-2. 